\title{
DEVELOPING A MONITORING SYSTEM FOR LONG-DISTANCE PIPELINE LEAKAGE INCORPORATING FUSION OF CONFLICTING EVIDENCES
}

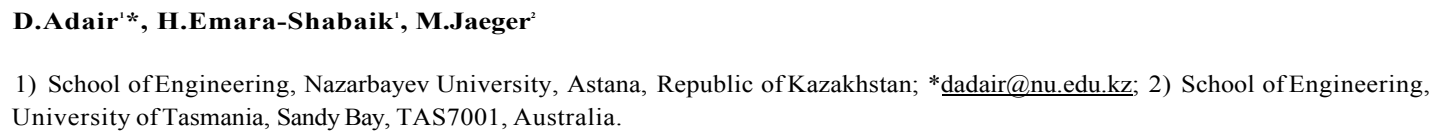

Introduction. When transporting petroleum products, the use of long-distance pipelines have become commonplace, due to their inherent efficiency and economy. However, such pipe systems often leak due to corrosion, natural destruction, and, natural aging and weathering, causing harm to the environment, fire etc. The ability to detect leakage with certainty and quickly is therefore very important. In this work, leakage is detected using a combination of a hydraulic method and Dezert-Smarandache theory.

Hydraulic method. A hydraulic model is used to determine a leakage by simulating its effects (i.e., for example, an increase in flow and drop in pressure) and compare them with values obtained from pressure and flow sensors upstream and downstream as shown.

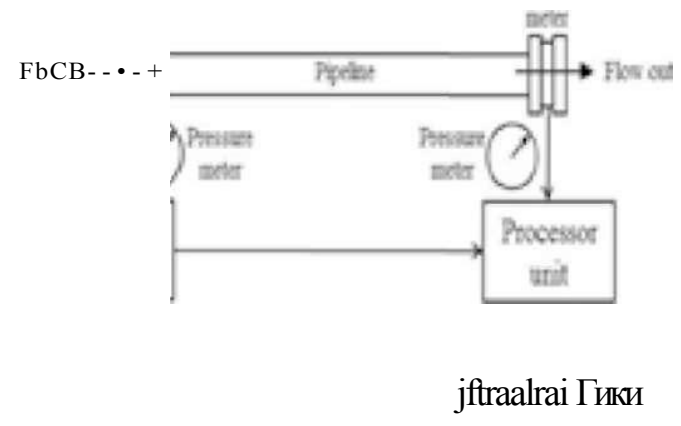

Each sensor records the waveform of a particular parameter and the corresponding processor unit classifies the waveform definite, i.e. true or false or uncertain. Before processing using the DezertSmarandache theory, a probability $\mathrm{m}(-)$ for belief in the classification is given.

Dezert-Smarandache theory. Key to providing quality decision options is an appropriate method of combining, or fusing, these heterogeneous evidence sources. Since the development of belief function theory introduced by Shafer in the 1970s many combination rules have been proposed in the literature because in highly conflicting situations the emblematic Dempster's rule generates counter-intuitive and unacceptable results in practical applications. In this work the Dezert-Smarandache theory (DSmT) will be explored, in particular the PCR5 and PCR6 rules of proportional conflict redistribution.

Conclusions. A decision algorithm which fuses evidence from pressure and flow meters has been developed for the detecting of long-distance oil pipeline leakage. The method of evidence fusion chosen here is the Dezert-Smarandache (DSmT) theory and in particular the Proportional Conflict Redistribution Rule nos. 5 and 6 (PCR5 and PCR6). 